\title{
General Inertia and Circle Criterion
}

\author{
Selim Solmaz ${ }^{* 1}$, Oliver Mason ${ }^{1}$, and Robert Shorten ${ }^{1}$ \\ ${ }^{1}$ Hamilton Institute, National University of Ireland-Maynooth, Co. Kildare, Ireland
}

\begin{abstract}
In this paper we extend the well known Kalman-Yacubovic-Popov (KYP) lemma to the case of matrices with general regular inertia. We show that the version of the lemma that was derived for the case of pairs of stable matrices whose rank difference is one, extends to the more general case of matrices with regular inertia and in companion form. We then use this result to derive an easily verifiable spectral condition for a pair of matrices with the same regular inertia to have a common Lyapunov solution (CLS), extending a recent result on CLS existence for pairs of Hurwitz matrices that can be considered as a time-domain interpretation of the famous circle criterion.
\end{abstract}

Copyright line will be provided by the publisher

\section{Introduction}

In recent years many publications $[1,2]$ have appeared that deal with the existence of common quadratic Lyapunov functions (CQLFs) for families of stable linear time-invariant (LTI) dynamical systems. Formally, for the case of a pair of systems, the CQLF existence problem amounts to determining necessary and sufficient conditions for the existence of a positive definite symmetric matrix $P=P^{T}>0, P \in \mathbb{R}^{n \times n}$ that simultaneously satisfies the matrix inequalities

$$
A_{1}^{T} P+P A_{1}<0 \quad, \quad A_{2}^{T} P+P A_{2}<0
$$

where $A_{1}, A_{2} \in \mathbb{R}^{n \times n}$ are Hurwitz. When there exists a $P=P^{T}>0$ satisfying the above inequalities, then the scalar function $V(x)=x^{T} P x$ is said to be a CQLF for the dynamical systems $\Sigma_{A_{i}}: \dot{x}=A_{i} x \quad i \in\{1,2\}$, and the matrix $P$ is a common Lyapunov solution (CLS) for the Lyapunov inequalities. The existence of CQLFs is of considerable importance in a number of engineering problems and consequently the CQLF existence problem has assumed a pivotal role in research on stability theory. Although it is often difficult to determine analytically the existence of a CQLF for a finite set of LTI systems, in certain situations elegant conditions for the existence of a CQLF may be obtained when restrictions are placed on the system matrices. Recently, one such result was obtained for a pair of Hurwitz matrices $A_{1}$ and $A_{2}$ with $\operatorname{rank}\left(A_{1}-A_{2}\right)=1$; in this case a CQLF exists for $\Sigma_{A_{1}}$ and $\Sigma_{A_{2}}$ if and only if the matrix product $A_{1} A_{2}$ does not have any real negative eigenvalues [3]. Furthermore, it has been shown in [1] that this result can be seen as a time-domain version of the Kalman-Yacubovich-Popov (KYP) lemma. The KYP lemma has played a key role in stability theory and has led to a number of important results on Lyapunov function existence for dynamical systems including the Circle Criterion [4]. In this paper, we shall extend the KYP lemma from classical stability theory to matrices with regular inertia and show that, in analogy with the classical case of Hurwitz matrices [4], this extension leads to elegant conditions for CLS existence for matrices with regular inertia also.

\section{Mathematical Preliminaries}

The inertia of a matrix $A \in \mathbb{R}^{n \times n}$ is the ordered triple $\operatorname{In}(A)=\left(i_{+}(A), i_{-}(A), i_{0}(A)\right)$ where $i_{+}(A), i_{-}(A), i_{0}(A)$ are the number of eigenvalues of $A$ in the open right half plane, the open left half plane, and on the imaginary axis, respectively. We say that $A$ has regular inertia if $i_{0}(A)=0$. We further denote by $\sigma_{\gamma[0, \infty)}\left[A_{1}, A_{2}\right]$ the parameterized family of matrices of the form $\left\{A_{1}+\gamma A_{2}: \gamma \in[0, \infty)\right\}$.

One of the most fundamental results on the stability of dynamical systems in the engineering literature is the Circle Criterion [4]. The relevance of the Circle Criterion in our present context stems from the fact that it provides a necessary and sufficient conditions for two fixed Hurwitz matrices in companion form to have a common Lyapunov solution. Formally, the result can be stated with the following theorem which follows from the single input single output (SISO) KYP lemma;

Theorem 2.1 $[1,3]$ Let two Hurwitz matrices $A, A-g h^{T}$ be given in $\mathbb{R}^{n \times n}$ with rank-1 difference, where g, $h$ are vectors in $\mathbb{R}^{n}$. Then a necessary and sufficient condition for the existence of a common Lyapunov solution for the matrices $A, A-g h^{T}$ is that

$$
1+\operatorname{Re}\left\{h^{T}(j \omega I-A)^{-1} g\right\}>0, \quad \forall \omega \in \mathbb{R} . \quad \text { (SISO Circle Criterion) }
$$

Moreover, the strict positive real condition (2) is equivalent to the matrix product $A\left(A-g h^{T}\right)$ having no negative real eigenvalues.

\footnotetext{
* Corresponding author: e-mail: selim.solmaz@nuim.ie, Phone: +353 1 7084536, Fax: +353 17086269
} 
The principal contribution of the present paper is to extend Theorem 2.1 to the case of pairs of matrices with the same regular inertia. First we recall some fundamental facts on the existence of solutions to the Lyapunov inequality for a single matrix with regular inertia.

Theorem 2.2 General Inertia Theorem [5]

Let $A \in \mathbb{R}^{n \times n}$ be given. Then there exists a symmetric matrix $P=P^{T}$ in $\mathbb{R}^{n \times n}$ such that $A^{T} P+P A<0$, if and only if $A$ has regular inertia. In this case, $\operatorname{In}(P)=\operatorname{In}(-A)$. Furthermore, if $\lambda_{i}+\lambda_{j} \neq 0$ for all eigenvalues $\lambda_{i}, \lambda_{j}$ of $A$, then for every negative definite $Q=Q^{T}<0$ in $\mathbb{R}^{n \times n}$, there is a unique $P=P^{T}$ with $\operatorname{In}(P)=\operatorname{In}(-A)$ and $A^{T} P+P A=Q<0$.

\section{Main results}

In this section we extend the classical Lefschetz [6] version of the KYP lemma to the case of companion matrices with regular inertia in Theorem 3.2. We then give a simple algebraic condition in Theorem 3.3 that is equivalent to CLS existence for a significant class of pairs of matrices in companion form, and with the same regular inertia. We shall first start with the statement of the following lemma that is instrumental in obtaining the extension of the KYP lemma in Theorem 3.2.

Lemma 3.1 [7] Let $A \in \mathbb{R}^{n \times n}$ be a nonsingular matrix such that for all pairs $\lambda_{i}, \lambda_{j}$ of eigenvalues of $A, \operatorname{Re}\left(\lambda_{i}+\lambda_{j}\right) \neq 0$. Further suppose that $g, h$ are column vectors in $\mathbb{R}^{n}$ such that for any $h$, the matrices $A$, and $A-g h^{T}$ can be transformed to companion forms using similarity transformations. Then $\operatorname{Re}\left\{h^{T}\left(j \omega I_{n}-A\right)^{-1} g\right\}=0, \forall \omega \in \mathbb{R}$ implies that $h=0$.

Making use of this lemma, and following Lefschetz's [6] way of proof, one can extent the classical KYP lemma to the case of matrices with regular inertia, which is given in the following theorem.

Theorem 3.2 [7] Let $A \in \mathbb{R}^{n \times n}$ be a companion matrix with regular inertia such that $\operatorname{Re}\left(\lambda_{i}+\lambda_{j}\right) \neq 0$ for all $\lambda_{i}, \lambda_{j} \in \sigma(A)$, and let $g, h \in \mathbb{R}^{n}$ be vectors such that $A-g h^{T}$ is also in companion form. Moreover, let $D=D^{T}>0$ in $\mathbb{R}^{n \times n}$ and $\tau>0$ in $\mathbb{R}$ be given. Then the following two statements are equivalent:

(i) There exists a symmetric matrix $P=P^{T}$ in $\mathbb{R}^{n \times n}$ with $\operatorname{In}(P)=\operatorname{In}(-A)$, a vector $q \in \mathbb{R}^{n}$ and a scalar $\varepsilon>0$ such that

$$
A^{T} P+P A=-q q^{T}-\varepsilon D, \quad \text { and } \quad P g-h=\sqrt{\tau} q
$$

(ii) $\tau+2 \operatorname{Re}\left\{h^{T}\left(j \omega I_{n}-A\right)^{-1} g\right\}>0$ for all $\omega \in \mathbb{R}$.

Now employing Theorem 3.2 we can obtain simple algebraic conditions for CLS existence for a significant class of pairs of matrices with the same regular inertia in $\mathbb{R}^{n \times n}$ which is stated in the following theorem.

Theorem 3.3 [7] Let $A, A-g h^{T}$ be two matrices in $\mathbb{R}^{n \times n}$ in companion form and with the same regular inertia, In $(A)=$ $\operatorname{In}\left(A-g h^{T}\right)=\left(n_{+}, n_{-}, 0\right)$, where $g, h$ are vectors in $\mathbb{R}^{n}$. Further, assume that for any pair of eigenvalues, $\lambda_{i}, \lambda_{j}$, of $A$, $\operatorname{Re}\left(\lambda_{i}+\lambda_{j}\right) \neq 0$. Then, the following statements are equivalent:

(i) There exists a matrix $P=P^{T}$ in $\mathbb{R}^{n \times n}$ with $\operatorname{In}(P)=\operatorname{In}(-A)$, and positive definite matrices $Q_{1}>0$, $Q_{2}>0$ such that

$$
A^{T} P+P A=-Q_{1}, \quad \text { and } \quad\left(A-g h^{T}\right)^{T} P+P\left(A-g h^{T}\right)=-Q_{2} .
$$

(ii) All matrices in the parameterized families $\sigma_{\gamma[0, \infty)}\left(A, A-g h^{T}\right)$ and $\sigma_{\gamma[0, \infty)}\left(A^{-1}, A-g h^{T}\right)$ have the same regular inertia.

(iii) The matrix $A\left(A-g h^{T}\right)$ has no real negative eigenvalues.

(iv) $1+\operatorname{Re}\left\{h^{T}\left(j \omega I_{n}-A\right)^{-1} g\right\}>0, \quad \forall \omega \in \mathbb{R}$.

Acknowledgements This work was partially supported by Science Foundation Ireland Grant 04/IN1/I478 and Science Foundation Ireland Grant 03/RPT1/I382. Science Foundation Ireland is not responsible for any use of data appearing in this publication.

\section{References}

[1] R.N. Shorten and K.S. Narendra, On common quadratic Lyapunov functions for pairs of stable LTI systems whose system matrices are in companion form, IEEE Transactions on Automatic Control, Vol.48, No.4, 618-621 (2003).

[2] N.Cohen and I.Lewkowicz, A pair of matrices sharing common Lyapunov solutions - a closer look, Linear Algebra and its Applications, Vol.360, 83-104 (2003).

[3] R.N. Shorten, O.Mason, F.O. Cairbre, and P.Curran, A unifying framework for the SISO Circle Criterion and other quadratic stability criteria, International Journal of Control, Vol.77, No.1, 1-8 (2004).

[4] K.S. Narendra and R.M. Goldwyn, A geometrical criterion for the stability of certain non-linear non-autonomous systems, IEEE Transactions on Circuit Theory, Vol.11, No.3, 406-407 (1964).

[5] R.Horn and C.Johnson, Topics in Matrix Analysis (Cambridge University Press, 1991).

[6] S.Lefschetz, Stability of Nonlinear Control Systems (Academic Press, 1965).

[7] O.Mason, R.Shorten, and S.Solmaz, On the Kalman-Yakubovich-Popov lemma and common Lyapunov solutions for matrices with regular inertia, Linear Algebra and its Aplications (2006). Accepted for publication. 